\title{
Sequential versus concurrent adjuvant chemo-endocrine therapy for HR+ early breast cancer: a systematic review and Bayesian network meta-analysis
}

\author{
Tianfu Li ${ }^{1,2 \#}$, Zhen Shan ${ }^{1 \#}$, Yawei Shi ${ }^{1}$, Xiaying Kuang ${ }^{1}$, Liang Yu ${ }^{1}$, Shou-Ching Tang ${ }^{3}$, Nan Shao ${ }^{1}$, \\ Ying $\operatorname{Lin}^{1}$ \\ ${ }^{1}$ Breast Disease Center, The First Affiliated Hospital, Sun Yat-sen University, Guangzhou, China; ${ }^{2}$ Laboratory of Surgery, The First Affiliated \\ Hospital, Sun Yat-sen University, Guangzhou, China; ${ }^{3}$ University of Mississippi Cancer Center and Research Institute, Jackson, MS, USA \\ Contributions: (I) Conception and design: Y Lin, N Shao; (II) Administrative support: Y Lin, Z Shan; (III) Provision of study materials or patients: \\ Liang Y; (IV) Collection and assembly of data: T Li, X Kuang, Y Shi; (V) Data analysis and interpretation: T Li, X Kuang, Y Shi; (VI) Manuscript \\ writing: All authors; (VII) Final approval of manuscript: All authors. \\ "These authors contributed equally to this work. \\ Correspondence to: Ying Lin; Nan Shao, MD, PhD. Breast Disease Center, The First Affiliated Hospital, Sun Yat-sen University, Guangzhou, 510080, \\ China. Email: linying3@mail.sysu.edu.cn; shaon@mail.sysu.edu.cn.
}

Background: Chemo-endocrine therapy is the standard adjuvant treatment strategy for hormone receptorpositive $(\mathrm{HR}+)$ early breast cancer. Our research aimed to compare the efficacy of adjuvant chemo-endocrine therapies, regarding different endocrinal regimens and integration sequences (sequential or concomitant), for $\mathrm{HR}+$ early breast cancer.

Methods: PubMed, Embase, the Cochrane Library and web of science were searched for articles published before October 2018 with Clinicaltrials.gov (https://clinicaltrials.gov) for registered clinical trials and ASCO, AACR, ESCO, SABCS meeting abstracts for addition. Randomized clinical trials (RCTs) comparing chemotherapy and/or endocrine therapy in the adjuvant treatment of primary breast cancer patients were included. Hazard ratios (HRs) of disease-free survival (DFS) and overall survival (OS) were extracted and analyzed in Bayesian analysis. Patients were stratified by menopause status.

Results: Thirty-three trials with 28,515 patients and 19 treatments were enrolled. Comparisons between regimens has seen better efficacy of ovarian function suppressor (OFS) + aromatase inhibitors (AI) than OFS + tamoxifen, either used concurrently [HR $=0.69,95 \%$ credible intervals (CrI): $0.47-1.02]$ or sequentially with chemotherapy ( $\mathrm{HR}=0.72,95 \% \mathrm{CrI}$ : 0.49-1.06) in premenopausal patients. Adding OFS to tamoxifen was marginally better than tamoxifen used alone (DFS: HR =0.85, 95\% CrI: 0.65-1.09; OS: HR =0.77, 95\% CrI: 0.52-1.08). Comparisons between different sequences of chemo-endocrine therapy proved equal efficacy in premenopausal and postmenopausal patients. Recommendation was given based on ranking of treatments. Sequential and concurrent use of chemotherapy and OFS + AI ranked equally in premenopausal patients and were recommended as the best option. However, tamoxifen ranked higher when used concurrently with chemotherapy in both premenopausal and postmenopausal HR+ early breast cancer.

Conclusions: In the adjuvant chemo-endocrine therapy for premenopausal HR+ early breast cancer, concurrent and sequential adjuvant chemo-endocrine therapy was demonstrated of equal efficacy in both postmenopausal and premenopausal HR+ early breast cancer.

Trial Registration: PROSPERO CRD42018104889.

Keywords: Estrogen receptor-positive breast cancer; chemotherapy; endocrine therapy; Bayesian analysis; randomized clinical trial (RCTs) 
Received: 04 March 2021; Accepted: 28 July 2021; Published: 31 January 2022.

doi: $10.21037 /$ tbcr-21-3

View this article at: https://dx.doi.org/10.21037/tbcr-21-3

\section{Introduction}

Breast cancer is the most common malignancy in women worldwide (1). Breast cancer treatment has seen evolutions over the years (2). Chemotherapy and endocrine therapy are demonstrated as the two major treatment strategies for hormone receptor-positive $(\mathrm{HR}+)$ early breast cancer $(2,3)$. Endocrine therapy is a routine treatment for $\mathrm{HR}+$ breast cancer, with most commonly used regimens categorized into: tamoxifen, aromatase inhibitors (AI) and ovarian function suppressor (OFS), among which, OFS was given to premenopausal patients to induce menopause status, while tamoxifen works as a selective estrogen receptor modulator by inhibiting estrogen-estrogen receptor binding and $\mathrm{AI}$ inhibiting androgens-estrogen conversion to decrease circulating estrogen level $(4,5)$. In the adjuvant setting, the combination of chemotherapy and endocrine therapy for HR+ early breast cancer had been demonstrated better efficacy than single regimens used alone, especially for women with median-high risk breast cancer, and significantly extended survival time (6-9). Chemotherapy has an early onset, therefore, contains generations of regimens. Systematic comparisons of chemotherapy were done between chemotherapy regimens of different generations in previously published meta-analysis, and demonstrated equal efficacy in $\mathrm{HR}+$ breast cancer. The largest difference in 5-year DFS for 3rd (anthracyclines and taxanes either concurrently or in sequence) $v s$. 2nd (anthracycline-based) generation is near $2.3 \%$ and even smaller when compared within generations (10). Paired comparisons between chemo-endocrine therapies had also been done in randomized clinical trials (RCTs) and traditional meta-analyses, however, considering the diversity of regimens used, systematic comparison of treatment schemes remained inconclusive (11).

Chemo-endocrine therapy includes not only the combination of regimens, but also the sequence of each treatment used. Clinical routines prefer the sequential use of chemo-endocrine therapy and clinical guidelines only based on limited evidence. By far, there are only 3 RCTs comparing concurrent versus sequential addition of tamoxifen to chemotherapy, however, all failed to reach statistical difference in either disease-free survival (DFS) or overall survival (OS) (12-14). Meta-analysis of these trials done by F. Poggio also found only a preference towards chemotherapy followed by tamoxifen [DFS: hazard ratio $(\mathrm{HR})=0.95$, 95\% CI: 0.76-1.18, $\mathrm{P}=0.643$; OS: $\mathrm{HR}=0.95$, 95\% CI: $0.80-1.12, \mathrm{P}=0.529]$ (15). The addition of AI to chemotherapy has also been sequentially used without any evidence from clinical trials. Comparison between different sequences for the integration of chemotherapy and $\mathrm{AI}$ is neglected. In recent years, RCTs like SOFT-TEXT have established the position of OFS in the adjuvant therapy of premenopausal HR+ early breast cancer $(16,17)$, the emergence of OFS + AI/TAM has further changed the blueprint of adjuvant endocrine therapy, especially for patients required adjuvant chemotherapy. The exploration of optimal timing for the integration of multi-regimens chemoendocrine therapy calls for tons of works in RCTs. However, further clinical trials in the adjuvant settings needs tedious time-consuming and rigorous conduction, therefore, can hardly be done, and the optimal timing for the integration of chemo-endocrine therapy remains unraveled.

Network meta-analysis (NMA) compares treatments both directly and indirectly within a directed acyclic graph (DAG), with direct comparisons based on RCTs and traditional meta-analysis so that indirect comparisons can be done between treatment arms without previous demonstration in RCT, therefore, NMA can simultaneously compare multiple therapies and provide novel insights between schemes without direct evidence from RCT (18). Moreover, Bayesian network analysis provides probabilities of each treatment on particular position among all treatments included in the DGA based on pooled therapeutic efficacy, as well as safety, cost, etc. if available and ranks treatments accordingly, thus providing recommendations for the selection of treatment from different perspectives.

The purpose of this study was to comprehensively compare the efficacy of chemo-endocrine therapies, regarding both different endocrine regimens and different integration sequences (sequential or concomitant), in the adjuvant therapy of $\mathrm{HR}+$ early breast cancer using Bayesian network analysis. We present the following article in accordance with the PRISMA reporting checklist (available at https://tbcr.amegroups.com/article/view/10.21037/tbcr$21-3 / \mathrm{rc})$. 


\section{Methods}

\section{Search strategy and selection criteria}

A systematic search was done in PubMed, Embase, the Cochrane Library and web of science for articles published; Clinicaltrials.gov (https://clinicaltrials.gov) for registered clinical trials; the American Society of Clinical Oncology (ASCO) annual meeting, the American Association for Cancer Research (AACR) annual meeting, the European Society for Medical Oncology (ESCO) annual meeting and the San Antonio Breast Cancer Symposium (SABCS) meeting for abstracts before July 2020. Previously published meta-analyses focusing on chemo-endocrine therapy of HR+ early breast cancer were searched for supplements (19). Searching terms were designed according to PICOS principles (20), detailed searching strategies was shown in Appendix 1 and PROSPERO registration profile (ID: CRD42018104889). Literature searches were performed by two researchers independently, with disagreement solved by a third researcher.

Eligible studies had to fulfill the following criteria: (I) English language published studies only; (II) RCTs compared the efficacy of adjuvant chemo-endocrine therapy in $\mathrm{HR}+$ early breast cancer patients with adjuvant chemoendocrine therapy used concomitantly or sequentially; (III) HRs of survival outcomes reported or extractable; (IV) patients with histologically diagnosed HR-positive early breast cancer without distant metastasis or second early tumor at diagnosis. Studies excluded from current analysis were those with the following characteristics: (I) non-RCTs; (II) non-HR+ breast cancer patients or HR+ subgroup not extractable; (III) RCTs with no arm treated with chemoendocrine therapy or patients previously treated with other pharmaceutical therapy; (IV) ongoing trials with no results; (V) researches published in other languages; (VI) HRs not reported or not extractable.

\section{Data collection and quality assessment}

The primary endpoint was DFS, and secondary endpoint were OS and relapse-free survival (RFS). DFS was defined as the time from randomization to either first relapse, second early tumor, or death without relapse, whichever occurred first. OS was defined as the time from randomization to death from any cause. RFS was defined as time from randomization to recurrence or contralateral breast cancer. For study reported only event free survival (EFS), defined as time from randomization to the first event of failure (locoregional recurrence, metastases, second primaries including contralateral breast cancer, or death), statistics were incorporated into DFS for analysis. HRs and $95 \%$ CIs were extracted and put into analyses (21). For literatures with only survival plots, HRs were generated using Engauge Digitizer version 10.11. For multi-arms trials, HRs were recalculated according to Jayne F Tierney' methods (22).

Study name, country, ethnicity, age, menopausal status, ER/PR status, surgical choices, interventions, sample size per group, randomization scheme, staging information, median follow-up with protocols were reviewed and extracted. Disparities were consoled with a third investigator for final decision. Treatments were extracted with both regimens and sequence. Quality of studies included were evaluated using the Cochrane risk-of-bias method and publication bias were evaluated using funnel diagram (23).

\section{Bayesian NMA}

Network plots of treatment arms enrolled were drawn using Stata/SE 15.1 software (StataCorp, College Station, Texas, USA). The network diagram consists of nodes and edges, representing the competing treatments and the available direct comparisons between pairs of treatments, respectively. Both nodes and edges were weighted according to the number of patients and studies enrolled.

NMA was conducted based on a Bayesian framework using OpenBUGS 3.2.3 software (MRC Biostatistics Unit, Cambridge, UK) (24) and "gemtc" package in RStudio Version 1.2. Deviance information criteria (DIC) was used to estimate model fitness, models with lower DIC were considered better fit (25). Random model was used in the following analyses (Table S1, Appendix 2).

The MCMC simulation were run for 50,000 burn-in iterations and 100,000 simulation iterations with 4 Markov chains. Extractions of values were done every 10 iterations. The convergence was assessed by trace plots and BrooksGelman-Rubin plots. The pooled estimates of HRs and 95\% credible intervals (CrIs) between two treatments were calculated. Based on the therapeutic efficacy, the probability of each treatment on each position was calculated and treatments were ranked by the position of the highest possibility. For two treatments with the highest possibility on the same position, the two treatments were considered a tied with equal preference.

Node-splitting analyses were applied to evaluate the consistency of models (26). The effects of comparisons 

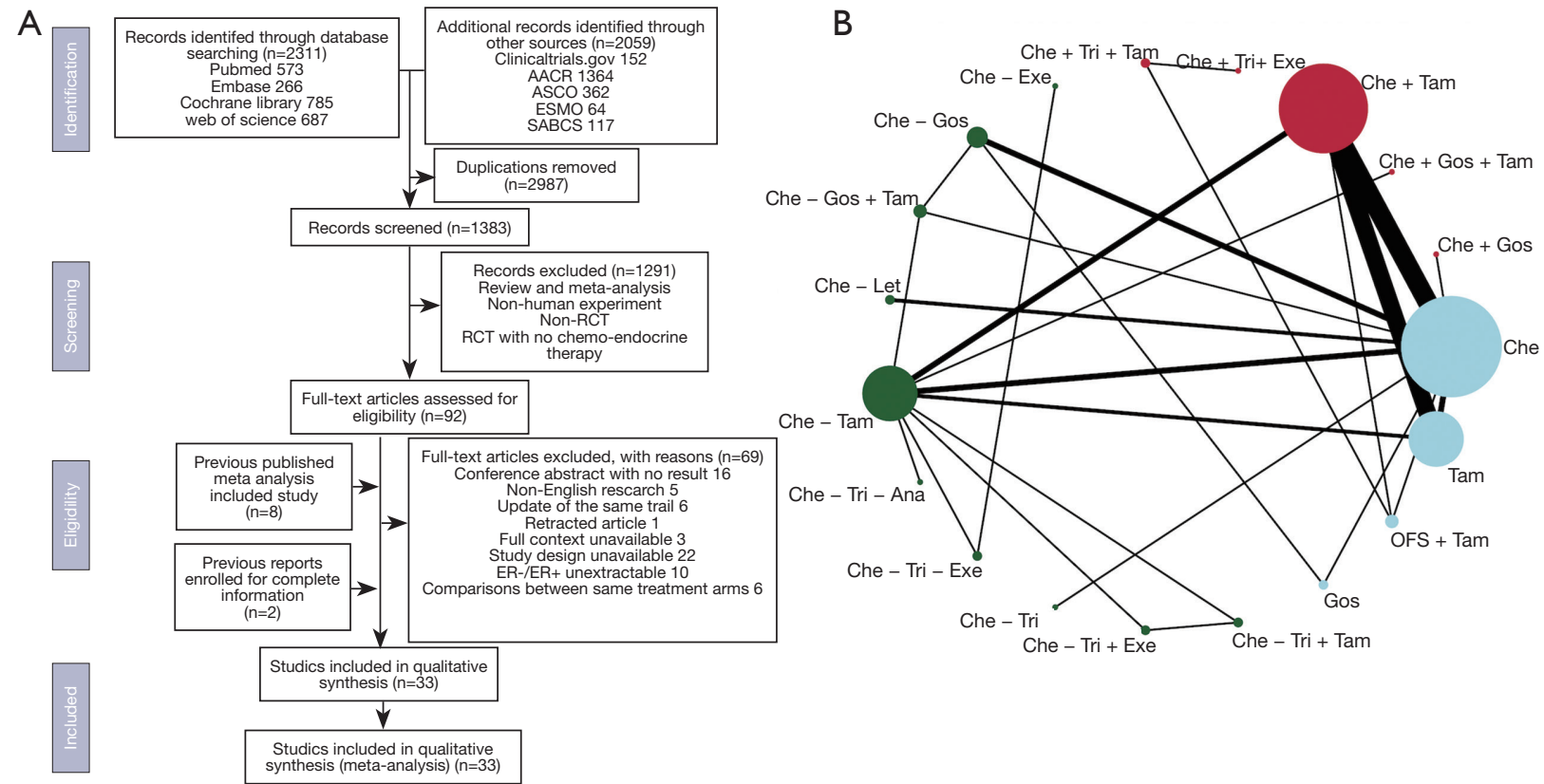

Figure 1 Workflow of literature selection and geometry of studies and treatment arms included. (A) Flow chart of literature search and selection process according to the PRISMA guidelines: (B) Network plots of studies and treatment arms enrolled. Nodes represent the competing treatments and edges represent the available direct comparisons between pairs of treatments. Both nodes and edges were weighted according to the number of patients and studies enrolled, respectively. Nodes were colored into three categories: chemotherapy or hormonal therapy alone (light blue); chemotherapy and endocrine therapy used concurrently (cranberry); chemotherapy followed by endocrine therapy (dark green). Che, chemotherapy; Tam, tamoxifen; OFS, ovarian function suppression; Tri, triptorelin; Gos, goserelin; Let, letrozole; Exe, exemestane; Ana, anastrozole. Sequence of regimens was illustrated as: sequential use, “-”; concurrent use, “+”.

in direct, indirect and network analysis were compared and different estimates $(\mathrm{P}<0.05)$ suggested the presence of inconsistency. Heterogeneity across studies was tested by $\mathrm{I}^{2}$ statistic: $\mathrm{I}^{2}=0 \%$ : no heterogeneity; $\mathrm{I}^{2}>25 \%$ : mild heterogeneity; $\mathrm{I}^{2}>50 \%$ : moderate heterogeneity; $\mathrm{I}^{2}>75 \%$ : high heterogeneity (27).

Given that chemotherapy regimens were previously demonstrated equal efficacy between generations in HR+ breast cancer (10), chemotherapy was not compared between regimens and recorded as with or without adjuvant chemotherapy only in this study. Analyses were done with patients stratified by menopausal status. Menopausal status was defined as pre-menopause and post-menopause. The Bayesian analysis was reported according to the Preferred Reporting Items for Systematic Reviews and Network Meta-Analysis (PRISMA-NMA) guidelines (28).

\section{Statistical analysis}

Statistical significance of comparisons between treatments was represented as $95 \% \mathrm{CrI}$ not cross 1 . Comparisons with $\mathrm{HR}<1$, upper $95 \% \mathrm{CrI} \leq 1.05$ or $\mathrm{HR}>1$, lower $95 \% \mathrm{CrI}$ $\geq 0.95$ were considered marginal significance. For all the other analyses without specific notification, a two-side $\mathrm{P}$ value $<0.05$ was considered significant.

\section{Results}

\section{Literature search and study characteristics}

The literature search left 1,383 literatures after removing duplication, among which 1,291 were removed due to literature type, species type, non-RCT or lack of chemoendocrine therapy arm. 102 studies were reviewed with full texts and a total of 33 studies were included for final analyses after thorough selection (Figure 1A). All studies included were evaluated for publication bias and risk of bias, all studies were demonstrated of low risks (Figure S1). The 33 studies included ranged from 1980 to 2020 with a total of 28,515 pathologically diagnosed female HR+ early breast cancer patients and 19 treatment arms (Figure 1B). Detailed 
characteristics of studies included were summarized in Table S2.

Chemotherapy was not compared between regimens. Endocrine therapy enrolled included tamoxifen, OFS and AI. OFS was achieved by bilateral oophorectomy, ovarian irradiation or luteinizing/gonadotrophin hormone-releasing hormone agonists (LHRHa/GnRHa), including triptorelin (Tri) and goserelin (Gos). Patients receiving Tri/Gos could subsequently undergo oophorectomy or irradiation. AI regimens included letrozole (Let), exemestane (Exe) and anastrozole (Ana). Sequences of regimens were defined as concurrent ("+": endocrine therapy concurrently used with chemotherapy) or sequential ("-": endocrine therapy sequentially used after chemotherapy). For treatments containing OFS + Tam/AI, the sequence of chemoendocrine therapy was defined based on the initiating timing of OFS used concurrently with or after chemotherapy.

For the three clinical outcomes enrolled, 23 studies contained DFS data between 18 treatment arms, 24 studies contained OS data between 13 treatment arms and only 8 studies contained RFS data between 6 treatments. Among all studies included, two trials reported EFS data, defined as time from randomization to the first event of failure (locoregional recurrence, metastases, second primaries including contralateral breast cancer, or death), which were identical with DFS defined in our study, thus were included in DFS analysis $(29,30)$.

Given the different clinical strategies between premenopausal and postmenopausal patients, comparisons were done with patients stratified by menopausal status. Studies with extractable subgroup endpoints were enrolled. Sixteen studies were included in premenopausal analysis and 18 studies in postmenopausal analysis. Geometry of studies and treatments included in each group were shown in Figure S2A,S2B. Four studies were excluded due to unextractable subgroup data (8,31-33). As for RFS analysis, analysis can be done in neither premenopausal nor postmenopausal group, therefore, analyses were done only regarding DFS and OS. Additionally, comparisons between OFS (triptorelin vs. goserelin) and AI (letrozole $v s$. exemestane $v s$. anastrozole) have seen no statistical significance, indicating equal efficacy within each category. Therefore, further comparisons were done with treatment arms merged by OFS and $\mathrm{AI}$ so as to explore for better significance (Figure S2C,S2D).

\section{Bayesian network analysis of chemo-endocrine therapy in premenopausal HR+ early breast cancer}

In premenopausal patients, comparisons between treatment arms found statistical significance only in DFS (Che Tam vs. Che - Tri + Exe: HR =1.61, 95\% CrI: 1-2.61; Che - Tri vs. Che - Tri + Exe: HR =2.17, 95\% CrI: $1.05-$ 4.58), but rarely in OS. Take marginal significance into account, further comparisons have seen Che - Gos + Tam significantly improved DFS compared to Che - Tri (HR $=0.6$, 95\% CrI: 0.33-1.05, Figure S3A).

With treatments merged by OFS and AI, comparisons still showed most statistical significances in DFS, with Che - OFS + AI demonstrated significant better efficacy than Che - Tam/OFS (Che - Tam: HR $=0.61,95 \%$ CrI: 0.41-0.9; Che - OFS: HR $=1.94,95 \%$ CrI: $1.22-3.11$ and Che - OFS + Tam significantly better than Che - OFS (HR =1.4, 95\% CrI: 1.02-1.92) (Figure 2A). Moreover, OFS + AI was demonstrated marginally better efficacy in $\mathrm{HR}+$ breast cancer than OFS + tamoxifen, either used concurrently with chemotherapy ( $\mathrm{HR}=0.69,95 \%$ CrI: $0.47-1.02)$ or sequentially after (HR $=0.72,95 \%$ CrI: $0.49-1.06)$ and the addition of OFS to tamoxifen was marginally better than tamoxifen used alone regarding both DFS (HR $=0.85,95 \%$ CrI: $0.65-1.09)$ and OS (HR $=0.77$, 95\% CrI: 0.52-1.08).

Comparisons between concurrent and sequential combination of chemo-endocrine therapy were done between Che +/- Tam, Che +/- OFS, Che +/- OFS + AI and Che +/- OFS + Tam. However, all comparisons were not statistical significance regarding either DFS or OS, indicating equal efficacy between sequences. Among all four comparisons, only Che +/- Tam were demonstrated slightly better efficacy when used concurrently regarding both DFS (HR $=0.86,95 \%$ CrI: $0.63-1.18)$ and OS (HR $=0.94,95 \%$ CrI: 0.67-1.31), while Che +/- OFS + Tam slightly better efficacy when used sequentially (DFS: $\mathrm{HR}=1.11,95 \% \mathrm{CrI}$ : 0.68-1.84); OS: HR $=1.21,95 \%$ CrI: 0.42-3.55).

Furthermore, with treatments ranked by efficacy, comparisons between concurrent and sequential combination of chemo-endocrine therapy were done based on ranking positions. With treatments merged by OFS and $\mathrm{AI}$, equal positions were seen in Che +/- OFS + AI and Che +/- OFS + Tam regarding both DFS and OS, with both Che +/- OFS + AI recommended as the best option 


\begin{tabular}{|c|c|c|c|c|c|c|c|c|c|c|c|}
\hline Che & & $\begin{array}{c}1.76 \\
(0.58,5.3)\end{array}$ & $\begin{array}{c}1.3 \\
(0.47,3.52)\end{array}$ & $\begin{array}{c}1.28 \\
(1.03,1.6) \\
\end{array}$ & $\begin{array}{c}1.19 \\
(0.84,1.7)\end{array}$ & $\begin{array}{c}1.63 \\
(0.96,2.79)\end{array}$ & $\frac{1.57}{(1.12,2.3)}$ & $\begin{array}{c}1.21 \\
(0.87,1.65)\end{array}$ & $\begin{array}{c}0.81 \\
(0.49,1.35)\end{array}$ & $\begin{array}{c}1.26 \\
(0.77,2.09)\end{array}$ & $\begin{array}{c}0.97 \\
(0.66,1.39)\end{array}$ \\
\hline $\begin{array}{c}1.28 \\
.79,2.09)\end{array}$ & he + OFS & & & & & & & & & & \\
\hline$\frac{2.17}{(1.15,4.15)}$ & $\begin{array}{c}1.69 \\
(0.77,3.79)\end{array}$ & $\begin{array}{c}\text { Che }+ \text { OFS } \\
+\mathrm{Al}\end{array}$ & $\begin{array}{c}0.74 \\
(0.46,1.2)\end{array}$ & $\begin{array}{c}0.73 \\
(0.24,2.21)\end{array}$ & $\begin{array}{c}0.68 \\
(0.21,2.16)\end{array}$ & $\begin{array}{c}0.93 \\
(0.27,3.14)\end{array}$ & $\begin{array}{c}0.9 \\
(0.28,2.89)\end{array}$ & $\begin{array}{c}0.69 \\
(0.22,2.15)\end{array}$ & $\begin{array}{c}0.46 \\
(0.14,1.55)\end{array}$ & $\begin{array}{c}0.72 \\
(0.27,1.91)\end{array}$ & $\begin{array}{c}0.55 \\
(0.17,1.74)\end{array}$ \\
\hline $\begin{array}{c}1.5 \\
(0.9,2.51)\end{array}$ & $\begin{array}{c}1.17 \\
(0.58,2.38)\end{array}$ & $\begin{array}{c}0.69 \\
(0.47,1.02)\end{array}$ & $\begin{array}{c}\text { Che + } \\
\text { OFS + Tam }\end{array}$ & $\begin{array}{c}0.98 \\
(0.36,2.68)\end{array}$ & $\begin{array}{c}0.92 \\
(0.32,2.68)\end{array}$ & $\begin{array}{c}1.25 \\
(0.4,3.87)\end{array}$ & $\begin{array}{c}1.21 \\
(0.42,3.55)\end{array}$ & $\begin{array}{c}0.93 \\
(0.33,2.67)\end{array}$ & $\begin{array}{c}0.62 \\
(0.2,1.92)\end{array}$ & $\begin{array}{c}0.97 \\
(0.41,2.3)\end{array}$ & $\begin{array}{c}0.74 \\
(0.26,2.15)\end{array}$ \\
\hline $\begin{array}{c}1.63 \\
(1.24,2.18)\end{array}$ & $\begin{array}{c}1.27 \\
(0.73,2.26)\end{array}$ & $\begin{array}{c}0.75 \\
(0.39,1.46)\end{array}$ & $\begin{array}{c}1.09 \\
(0.64,1.86)\end{array}$ & Che + Tam & $\begin{array}{c}0.93 \\
(0.62,1.4)\end{array}$ & $\begin{array}{c}1.28 \\
(0.73,2.21)\end{array}$ & $\begin{array}{c}1.23 \\
(0.84,1.86)\end{array}$ & $\begin{array}{c}0.94 \\
(0.67,1.31)\end{array}$ & $\begin{array}{c}0.63 \\
(0.37,1.1)\end{array}$ & $\begin{array}{c}0.98 \\
(0.59,1.65)\end{array}$ & $\begin{array}{c}0.76 \\
(0.451,1.08)\end{array}$ \\
\hline $\begin{array}{c}1.19 \\
(0.97,1.48)\end{array}$ & $\begin{array}{c}0.93 \\
(0.55,1.59)\end{array}$ & $\begin{array}{c}0.55 \\
(0.28,1.06)\end{array}$ & $\begin{array}{c}0.8 \\
(0.47,1.36)\end{array}$ & $\begin{array}{c}0.73 \\
(0.52,1.03)\end{array}$ & Che - OFS & $\begin{array}{c}1.37 \\
(0.75,2.48)\end{array}$ & $\begin{array}{c}1.32 \\
(0.89,2.02)\end{array}$ & $\begin{array}{c}1.01 \\
(0.65,1.56)\end{array}$ & $\begin{array}{c}0.68 \\
(0.4,1.14)\end{array}$ & $\begin{array}{c}1.05 \\
(0.57,1.94)\end{array}$ & $\begin{array}{c}0.81 \\
(0.48,1.33)\end{array}$ \\
\hline$\frac{2.31}{(1.5,3.64)}$ & $\begin{array}{c}1.8 \\
(0.94,3.52)\end{array}$ & $\begin{array}{c}1.07 \\
(0.53,2.14)\end{array}$ & $\begin{array}{c}1.54 \\
(0.86,2.77)\end{array}$ & $\begin{array}{c}1.42 \\
(0.87,2.3)\end{array}$ & $\frac{1.94}{(1.22,3.11)}$ & $\begin{array}{c}\text { Che - OFS } \\
+\mathrm{Al}\end{array}$ & $\begin{array}{c}0.96 \\
(0.59,1.61)\end{array}$ & $\begin{array}{c}0.74 \\
(0.45,1.18)\end{array}$ & $\begin{array}{c}0.49 \\
(0.24,1.03)\end{array}$ & $\begin{array}{c}0.77 \\
(0.37,1.6)\end{array}$ & $\begin{array}{c}0.59 \\
(0.31,1.12)\end{array}$ \\
\hline$\frac{1.66}{(1.26,2.24)}$ & $\begin{array}{c}1.3 \\
(0.74,2.3)\end{array}$ & $\begin{array}{c}0.77 \\
(0.41,1.45)\end{array}$ & $\begin{array}{c}1.11 \\
(0.68,1.84)\end{array}$ & $\begin{array}{c}1.02 \\
(0.71,1.46)\end{array}$ & $\stackrel{1.4}{(1.02,1.92)}$ & $\begin{array}{c}0.72 \\
(0.49,1.06)\end{array}$ & $\begin{array}{c}\text { Che - } \\
\text { OFS + Tam }\end{array}$ & $\begin{array}{c}0.77 \\
(0.52,1.08)\end{array}$ & $\begin{array}{c}\underline{0.51} \\
(0.28,0.92) \\
\end{array}$ & $\begin{array}{c}0.8 \\
(0.43,1.45)\end{array}$ & $\begin{array}{c}0.62 \\
(0.36,1)\end{array}$ \\
\hline$\frac{1.41}{(1.07,1.86)}$ & $\begin{array}{c}1.1 \\
(0.63,1.93)\end{array}$ & $\begin{array}{c}0.65 \\
(0.36,1.15)\end{array}$ & $\begin{array}{c}0.94 \\
(0.61,1.44)\end{array}$ & $\begin{array}{c}0.86 \\
(0.63,1.18)\end{array}$ & $\begin{array}{c}1.18 \\
(0.86,1.63)\end{array}$ & $\frac{0.61}{(0.41,0.9)}$ & $\begin{array}{c}0.85 \\
(0.65,1.09)\end{array}$ & Che - Tam & $\begin{array}{c}0.67 \\
(0.37,1.21)\end{array}$ & $\begin{array}{c}1.04 \\
(0.59,1.88)\end{array}$ & $\begin{array}{c}0.8 \\
(0.5,1.27)\end{array}$ \\
\hline $\begin{array}{c}0.94 \\
(0.65,1.37)\end{array}$ & $\begin{array}{c}0.73 \\
(0.4,1.36)\end{array}$ & $\frac{0.43}{(0.21,0.9)}$ & $\begin{array}{c}0.62 \\
(0.33,1.17)\end{array}$ & $\begin{array}{c}0.57 \\
(0.36,0.96) \\
\end{array}$ & $\begin{array}{c}0.79 \\
(0.53,1.15)\end{array}$ & $\frac{0.41}{(0.23,0.72)}$ & $\begin{array}{l}\underline{0.56} \\
(0.35,0.88) \\
\end{array}$ & $\begin{array}{c}0.67 \\
(0.42,1.05)\end{array}$ & $\underline{\text { OFS }}$ & $\begin{array}{c}1.4 \\
(0.59,3.18)\end{array}$ & $\begin{array}{c}1.2 \\
(0.63,2.21)\end{array}$ \\
\hline $\begin{array}{c}1.53 \\
(0.68,3.49)\end{array}$ & $\begin{array}{c}1.19 \\
(0.46,3.11)\end{array}$ & $\begin{array}{c}0.71 \\
(0.33,1.5)\end{array}$ & $\begin{array}{c}1.02 \\
(0.53,1.94)\end{array}$ & $\begin{array}{c}0.94 \\
(0.41,2.16)\end{array}$ & $\begin{array}{c}1.28 \\
(0.55,2.97)\end{array}$ & $\begin{array}{c}0.66 \\
(0.28,1.57)\end{array}$ & $\begin{array}{c}0.92 \\
(0.41,2.07)\end{array}$ & $\begin{array}{c}1.09 \\
(0.5,2.36)\end{array}$ & $\begin{array}{c}1.63 \\
(0.66,4.03)\end{array}$ & $\mathrm{FS}+$ Tam & $\begin{array}{c}0.77 \\
(0.41,1.39)\end{array}$ \\
\hline$\frac{1.44}{(1.04,2.01)}$ & $\begin{array}{c}1.12 \\
(0.63,2.04)\end{array}$ & $\begin{array}{c}0.66 \\
(0.33,1.32)\end{array}$ & $\begin{array}{c}0.96 \\
(0.54,1.71)\end{array}$ & $\begin{array}{c}0.88 \\
(0.66,1.16)\end{array}$ & $\begin{array}{c}1.21 \\
(0.82,1.77)\end{array}$ & $\begin{array}{c}0.62 \\
(0.37,1.05)\end{array}$ & $\begin{array}{c}0.86 \\
(0.57,1.3)\end{array}$ & $\begin{array}{c}1.02 \\
(0.7,1.5)\end{array}$ & $\begin{array}{c}1.53 \\
(0.93,2.54)\end{array}$ & $\begin{array}{c}0.94 \\
(0.4,2.23)\end{array}$ & $\underline{\text { Tam }}$ \\
\hline
\end{tabular}

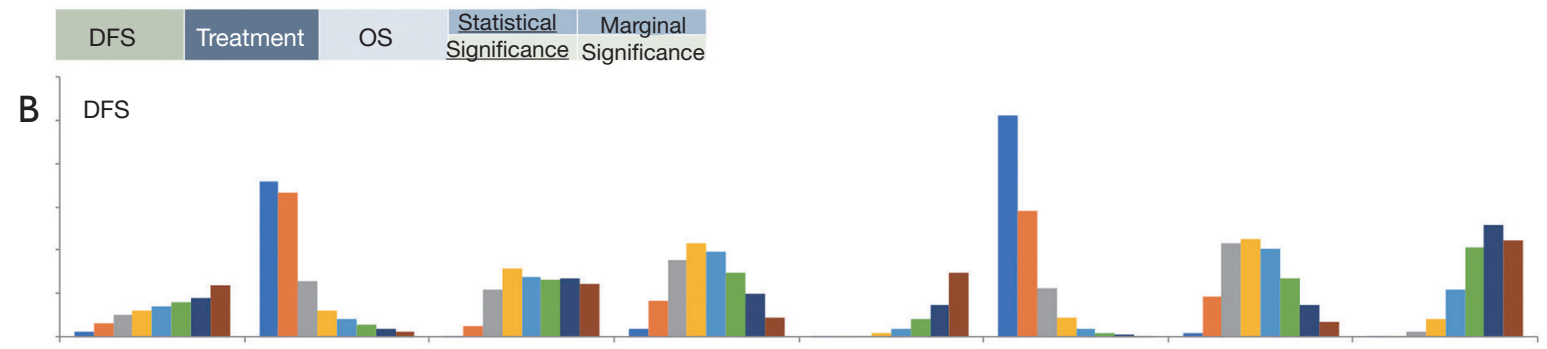

$\mathrm{Che}+\mathrm{OFS}$ Che $+\mathrm{OFS}+\mathrm{AlChe}+\mathrm{OFS}+\mathrm{Tam} \mathrm{Che}+\mathrm{Tam}$

Che - OFS Che - OFS + Al Che - OFS + Tam Che - Tam

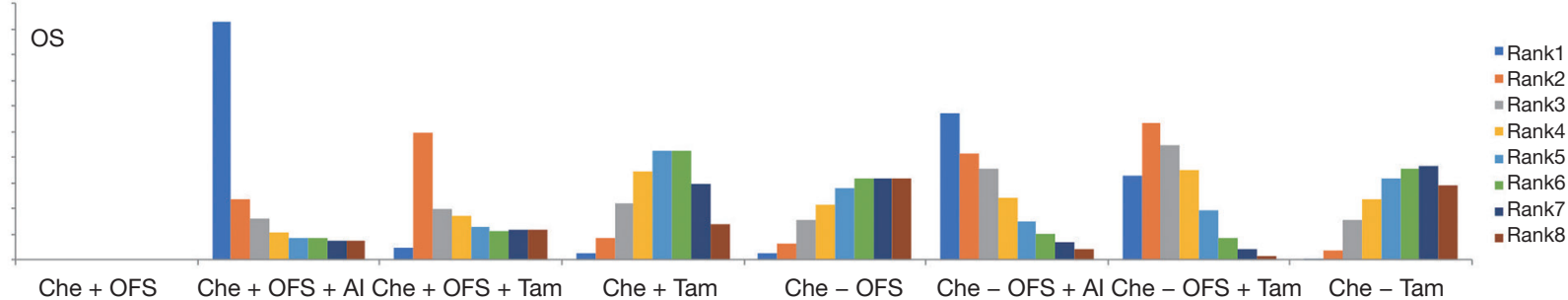

Figure 2 Comparisons of chemo-endocrine therapies for premenopausal HR+ early breast cancer regarding DFS, OS. (A) Comparisons of chemo-endocrine therapy for premenopausal HR+ early breast cancer by Bayesian network analysis regarding DFS and OS. Treatment arms were aligned on the diagonal line. Hazard ratios and $95 \%$ CrIs were generated by comparing the upper-left arm to the lower-right. Bricks were colored by clinical outcomes: DFS: light green, OS: light blue. 95\% CrI not cross 1 was considered statistically significant and represented as bold deep green (DFS) or deep blue (OS) bricks with underline. HR $<1$, upper $95 \% \mathrm{CrI} \leq 1.05$ and HR $>1$, lower $95 \%$ $\mathrm{CrI} \geq 0.95$ were considered marginal significance and represented as deep green (DFS) or deep blue (OS) bricks only. (B) Rankogram of treatment arms in DFS and OS analysis for premenopausal HR+ early breast cancer. Based on the therapeutic efficacy, the probability of each treatment on each position was calculated and presented as bar plot. Treatments were ranked by the position of the highest possibility. For two treatments with the highest possibility on the same position, the two treatments were considered a tied with equal preference. DFS, diseases-free survival; OS, overall survival; CrIs, credible intervals; Che, chemotherapy; Tam, tamoxifen; OFS, ovarian function suppression; $\mathrm{AI}$, aromatase inhibitor. 
and Che +/- OFS + Tam as the second. Meanwhile, in both DFS and OS, the concurrent use of chemotherapy and tamoxifen ranked higher than used sequentially, indicating putative benefit of Che + Tam in premenopausal HR+ early breast cancer (Figure 2B, Figure S3B).

\section{Bayesian network analysis of chemo-endocrine therapy in postmenopausal HR+ early breast cancer}

In postmenopausal patients, comparisons were done between 8 treatments in DFS analysis and 5 treatments in OS analysis. However, no statistical significance was seen in either DFS or OS (Figure S3C). Further analyses with treatments merged by AI have seen the identical pattern, with most statistical significances seen in DFS, demonstrating the therapeutic effect of adjuvant chemoendocrine therapy in improving the DFS of not only premenopausal, but also postmenopausal $\mathrm{HR}+$ breast cancer (Figure 3A). Moreover, comparisons between regimens demonstrated the addition of $\mathrm{AI}$ after tamoxifen significantly improved DFS (Che + Tam vs. Che - TamAI: $\mathrm{HR}=1.59,95 \%$ CrI: 1.01-2.61; Che + Tam vs. Che Tam-AI: HR $=1.54,95 \%$ CrI: $1-2.44$ ), however, the clinical advantage of AI over tamoxifen was not seen (Che + Tam vs. Che - AI: HR =1.36, 95\% CrI: 0.85-2.13; Che - AI vs. Che - Tam: HR $=0.76,95 \%$ CrI: $0.48-1.23$ ), so as the comparison between Che +/- Tam regarding both DFS and OS (DFS: HR $=1.03,95 \%$ CrI: $0.8-1.35$; OS: HR $=0.91$, 95\% CrI: $0.73-1.15)$.

Treatments recommended for postmenopausal patients highlighted the therapeutic efficacy of AI in the adjuvant chemo-endocrine therapy for postmenopausal HR+ early breast cancer, with Che - Tam-AI and Che - AI recommended as the best and second in DFS, respectively, and $\mathrm{Che}-\mathrm{AI}$ recommended as the best for OS. Comparisons between Che +/- Tam based on the ranking position has seen the identical position in DFS but a higher position when used concurrently in OS (Figure 3B). Comparatively, similar results were seen in the ranking of treatments not merged (Figure S3D).

\section{Evaluation of inconsistency and heterogeneity}

Evaluation of inconsistency were done in all comparisons. In DFS analyses between sequential and concurrent use of chemotherapy and tamoxifen, inconsistency was only seen in premenopausal patients (Figure S4A,S4B), but not in OS analysis (Figure S5A,S5B). In premenopausal patients, comparisons between sequential and concurrent integration of chemotherapy and tamoxifen showed disagreement between direct and indirect comparison $(\mathrm{P}=0.0394)$. Despite insignificance in traditional metaanalysis, indirect comparisons showed better efficacy of concurrent use. However, network analysis revealed no difference with combined consideration of the two. This showed putative subgroups with different clinical response using chemotherapy and tamoxifen.

In premenopausal patients, heterogeneity was mainly seen in direct and indirect comparisons of DFS between Che +/- Tam. Except for this, comparison between Che Tam with/without AI showed heterogeneity in DFS analysis that came from direct and indirect comparisons $\left(\mathrm{I}^{2}=65.4 \%\right)$ in postmenopausal patients (Figure S6A,S6B).

Comparison between Che - Tam with/without OFS showed heterogeneity in OS analysis that also came from direct and indirect comparisons in premenopausal patients $\left(\mathrm{I}^{2}=41.2 \%\right)$. In postmenopausal patients, heterogeneity was seen in comparisons between sequential and concurrent use of chemotherapy and tamoxifen with heterogeneity mainly came from pair-wised comparisons of RCTs (Figure S7A,S7B).

\section{Discussion}

Comprehensive comparison of chemo-endocrine therapy required integrative evaluations focusing on both different chemo-endocrine regimens and different sequences for integration, which remained unraveled. By adopting Bayesian network analysis, our work aimed to compare different endocrine regimens and different integration chemo-endocrine sequences in adjuvant setting of HR+ early breast cancer comprehensively. After systematic review of RCTs, 33 trials with 28,515 pathologically diagnosed female HR+ early breast cancer patients were enrolled. Comparisons between different sequences of chemo-endocrine therapy proved equal efficacy, either used sequentially or concurrently, in both premenopausal and postmenopausal patients. The ranking of treatments generated by Bayesian network analysis has also seen equal recommendations of Che +/- OFS + AI and Che +/- OFS + Tam regarding both DFS and OS in premenopausal patients. Intriguingly, only tamoxifen ranked higher when used concurrently with chemotherapy in both premenopausal and postmenopausal patients, indicating putative benefit of Che + Tam in HR+ early breast cancer.

In premenopausal patients, comparisons between endocrine regimens have seen better efficacy of OFS + AI 

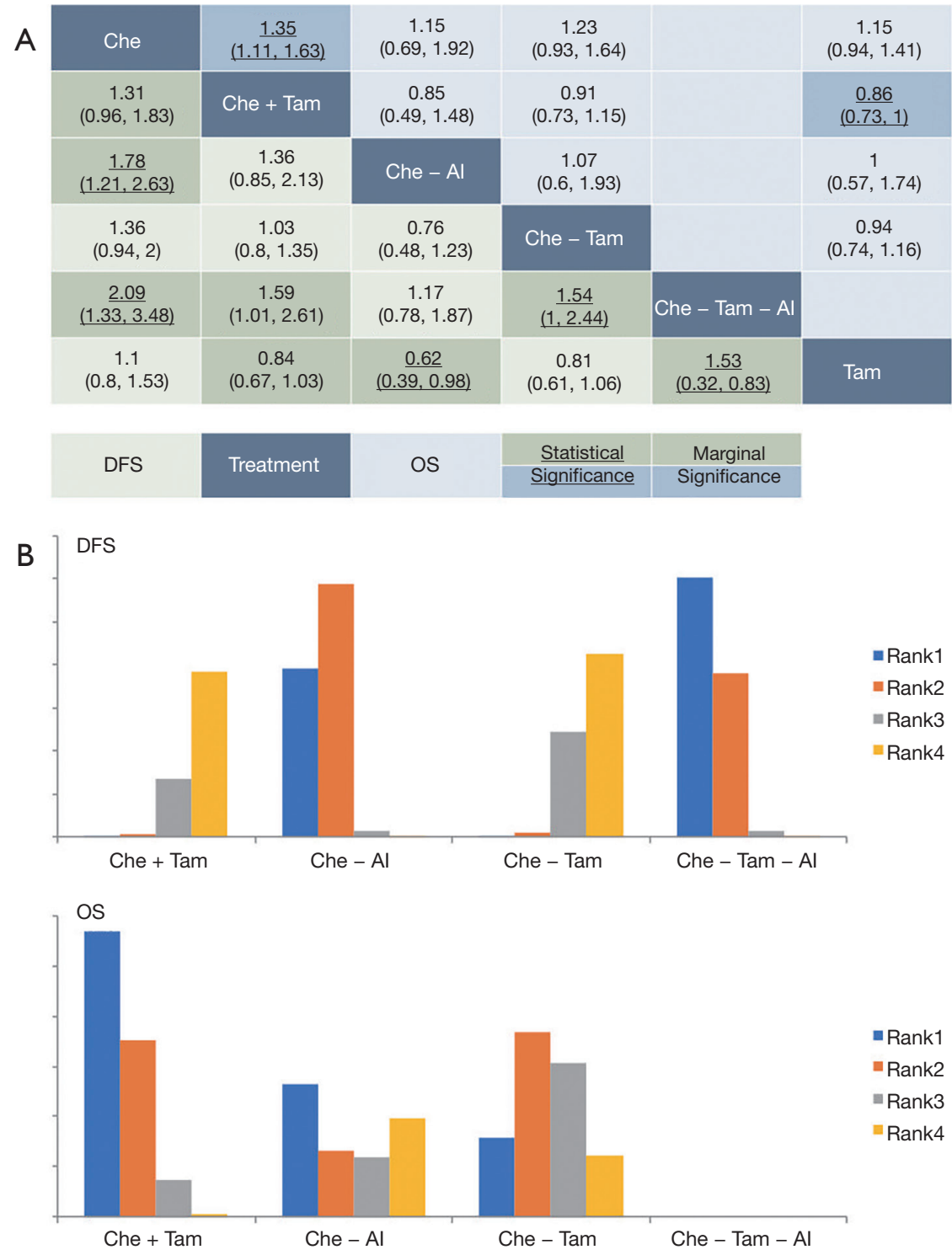

Figure 3 Comparisons of chemo-endocrine therapies for postmenopausal HR+ early breast cancer regarding DFS, OS. (A) Comparisons of chemo-endocrine therapy for postmenopausal HR+ early breast cancer by Bayesian network analysis regarding DFS and OS. (B) Rankogram of treatment arms in DFS and OS analysis for postmenopausal HR+ early breast cancer. DFS, diseases-free survival; OS, overall survival.

than OFS + tamoxifen in improving DFS, as well as Adding OFS to tamoxifen compared to tamoxifen alone. These results are in accordance with data from SOFT/TEXT (16) and ASTRRA (34). The introduction of OFS in the adjuvant chemo-endocrine therapy caused discussions focusing on the initiating time of OFS and chemotherapy. In our study, both sequentially or concurrently used chemotherapy and OFS $+\mathrm{AI} /$ Tam ranked equally as the best and second options in premenopausal patients, respectively. The integrative analysis of TEXT and SOFT showed a similar result, in which the concurrent use of triptorelin with chemotherapy demonstrated a not significant difference in BCFI compared with sequential triptorelin after chemotherapy ( $\mathrm{HR}=1.11$, 95\% CI: $0.72-1.72, \mathrm{P}=0.72$ ). However, analysis of DFS and OS cannot be done due to guarantee-time bias (35). By adopting Bayesian network analysis with data extracted from TEXT and SOFT, respectively, DFS and OS were firstly compared between Che +/- Tri + Exe and Che +/- 
Tri + Tam in our study, which providing novel insight into the exploration of optimal timing for the integration of OFS and chemotherapy.

Current researches found significant improvement in the survival of advanced breast cancer patients treated with chemotherapy alongside with AI or fulvestrant comparing to either chemotherapy or endocrine therapy used alone (36-39). Albeit the indirect comparisons between concurrent and sequential use of the same agents, increased response rate and response durations rendered indicating putative additive and synergistic effects of AI or fulvestrant used with chemotherapy, which is different to the antagonistic effect between tamoxifen and cytotoxic reported (40). Furthermore, similar comparisons were also explored in neoadjuvant therapy. The addition of letrozole concurrently with neoadjuvant chemotherapy was demonstrated a prominent clinical and pathologic response rates compared to chemotherapy alone in postmenopausal HR+ locally advanced breast cancer (41). Latest update of CBCSG-036 reported an improvement of tumor overall response rates in ER-positive, HER2-negative breast cancer patients treated with neoadjuvant concurrent chemo-endocrine therapy (ORR: $84.8 \%$ vs. $72.6 \%$, odds ratio $=2.11,95 \%$ CI: $1.13-3.95, \mathrm{P}=0.02$, pCR: $7.2 \%$ vs. $4.0 \%, \mathrm{P}=0.278$ ) (42), demonstrated the synergistic effect with concurrent chemo-endocrine therapy in neoadjuvant setting. These evidences raised challenges to the sequential administration of chemotherapy and endocrine therapy in the adjuvant settings of HR+ breast cancer.

Comparisons between concurrent and sequential combination of tamoxifen to chemotherapy were done in RCTs and no significant difference was seen. In GEICAM 9401 study, comparisons were done in postmenopausal node-positive breast cancer patients, DFS at 5 years was $70 \%$ in the concurrent group and $75 \%$ in the sequential group (HR $=1.11,95 \% \mathrm{CI}: 0.71-1.73, \mathrm{P}=0.64)(12)$. In SWOG-8814, insignificant differences was showed regarding both DFS and OS in postmenopausal nodepositive $\mathrm{HR}+$ breast cancer patients, favoring $\mathrm{CHE}$ seq TAM (DFS: HR =0.84, 95\% CI: 0.70-1.01, $\mathrm{P}=0.061$; OS: HR $=0.90,95 \%$ CI: 0.73-1.10, P=0.30) (13). Bedognetti et al. compared the efficacy of concurrent $v s$. sequential administration of adjuvant chemotherapy and tamoxifen in node-positive primary breast cancer but also found no difference between concurrent or sequential use, subgroup analysis in HR+ patients showed no difference (HR of relapse $=1.16,95 \%$ CI: $0.88-1.52, \mathrm{P}=0.36$; HR of death $=1.06,95 \%$ CI: $0.78-1.44, \mathrm{P}=0.76$ ) (14). Despite the insignificances, all comparisons revealed the sequential combination of tamoxifen and chemotherapy of slight better efficacy, which were further demonstrated in a previously reported meta-analysis (DFS: HR $=0.95,95 \%$ CI: $0.76-1.18, \mathrm{P}=0.643$; OS: $\mathrm{HR}=0.95,95 \%$ CI: 0.80 $1.12, \mathrm{P}=0.529$ ) (15). However, our results indicated the putative advantages of tamoxifen concurrently used with chemotherapy in HR+ early breast cancer. Comparatively, a collaborative meta-analysis of individual patient data from 20 trials $(n=21,457)$ comparing the 5 years of tamoxifen versus no adjuvant tamoxifen in early breast cancer was done by Early Breast Cancer Trialists' Collaborative Group (EBCTCG). Subgroup analysis demonstrated tamoxifen was of slight superiority of recurrence rate ratio $(R R)$ when starting concurrently with the chemotherapy $[R R=0.62$ (SE $=0.06)$ ] comparing to be used after it $[\mathrm{RR}=0.71(\mathrm{SE}=0.05)]$, as well as better death rate ratio [concurrent: $\mathrm{RR}=0.69$ $(\mathrm{SE}=0.08)$; sequential: $\mathrm{RR}=0.78(\mathrm{SE}=0.07)](43)$, which in accordance with our results. Preclinical studies regarding the combination of chemotherapy and tamoxifen were done. Sutherland et al. showed that Tamoxifen inhibited cell growth and increased the proportion of G0/G1 cells (40). Therefore, the anti-tumor activity of 5-fu and doxorubicin is weakened. However, another explanation supporting concurrent use chemo-endocrine therapy was proclaimed focusing on the impact of intra-tumor heterogeneity (44-46). In addition to the chemotherapy-responsive cells, there should be tumor cells that were highly responsive to endocrine therapy, such as cells with high levels of estrogen receptors (ER) and/or progesterone receptors $(\mathrm{PgR})$, co-exist, therefore, starting endocrine therapy after chemotherapy would be a delay to these cells and chemotherapy-induced tumor escape and migration were also found that threat the outcomes of breast cancer patients (47). Combining both studies on molecular level and clinical trials, the concurrent efficacy chemotherapy and tamoxifen requests further discussions.

Limitations were seen in this work, one of which is the coverage of endocrine regimens, especially for treatment with concurrent chemotherapy and AI. The significantly better efficacy of AI found in our study indicating the therapeutic efficacy of chemotherapy used with AI of utter importance, especially for postmenopausal patients. However, due to the absence of treatment arm using AI concurrently with chemotherapy, comparison cannot be done. Secondly, the absence of estimation and comparison focusing on the adverse side effects that may severely diminish the quality of life for breast cancer patients owing 
to the complexity of clinical trials enrolled. Adverse effects reported in studies included showed a huge diversity given the timespan as well as the study design, which made it impossible to compare the difference between each treatment arms using Bayesian analysis. However, there is little overlap of toxicities between chemotherapy and endocrine therapy. Furthermore, the toxicities from endocrine therapies are mostly minor and are less likely to affect the tolerability for chemotherapy. Therefore, the possible added toxicities from the combined chemoendocrine therapy should not deter the use of this modality, especially in premenopausal women who may benefit from a possible survival advantage.

Despite the limitations mentioned, significance should be seen in our study. Except for the advantages seen in AI compared to tamoxifen and combined use of OFS and Tam/ $\mathrm{AI}$ to Tam/AI alone, comparisons focusing on the sequence of chemo-endocrine therapy has seen chemotherapy recommended to be concurrently used with tamoxifen in both premenopausal and postmenopausal patients based on Bayesian ranking results. Therefore, we may reconsider the sequence of adjuvant chemo-endocrine therapy. Moreover, the significant better efficacy of AI found in our study indicating the therapeutic efficacy of chemotherapy used with AI of utter importance. GIM10-CONSENT (NCT02918084), the only ongoing RCT comparing concomitant $v s$. sequential administration of AIs with chemotherapy in the adjuvant therapy of postmenopausal early breast cancer patients, is still in recruiting but will provide initial estimation of outcomes in 2028, therefore, a future update should be expected.

In conclusion, comparisons between regimens in our study demonstrated that OFS + AI was of better efficacy than OFS + Tamoxifen and adding OFS to Tam significantly improved DFS in the adjuvant chemo-endocrine therapy of premenopausal HR+ early breast cancer. Comparisons between sequences revealed concurrent and sequential chemo-endocrine therapy of equal efficacy in both postmenopausal and premenopausal patients. However, Bayesian network ranking showed better DFS and OS with concurrent tamoxifen and chemotherapy than used sequentially for both postmenopausal and premenopausal patients.

\section{Acknowledgments}

Funding: The study was funded by Sun Yat-sen University Clinical Research 5010 Program 2016007.

\section{Footnote}

Reporting Checklist: The authors have completed the PRISMA reporting checklist. Available at https://tbcr. amegroups.com/article/view/10.21037/tbcr-21-3/rc

Conflicts of Interest: All authors have completed the ICMJE uniform disclosure form (available at https://tbcr. amegroups.com/article/view/10.21037/tbcr-21-3/coif). The authors have no conflicts of interest to declare.

Ethical Statement: The authors are accountable for all aspects of the work in ensuring that questions related to the accuracy or integrity of any part of the work are appropriately investigated and resolved.

Open Access Statement: This is an Open Access article distributed in accordance with the Creative Commons Attribution-NonCommercial-NoDerivs 4.0 International License (CC BY-NC-ND 4.0), which permits the noncommercial replication and distribution of the article with the strict proviso that no changes or edits are made and the original work is properly cited (including links to both the formal publication through the relevant DOI and the license). See: https://creativecommons.org/licenses/by-nc-nd/4.0/.

\section{References}

1. Bray F, Ferlay J, Soerjomataram I, et al. Global cancer statistics 2018: GLOBOCAN estimates of incidence and mortality worldwide for 36 cancers in 185 countries. CA Cancer J Clin 2018;68:394-424.

2. Zurrida S, Veronesi U. Milestones in breast cancer treatment. Breast J 2015;21:3-12.

3. Bychkovsky BL, Dizon DS, Sikov WM. Systemic Therapies for Nonmetastatic Breast Cancer: The Role of Neoadjuvant and Adjuvant Chemotherapy and the Use of Endocrine Therapy. Clin Obstet Gynecol 2016;59:756-71.

4. Neven P, Jongen L, Lintermans A, et al. Tamoxifen Metabolism and Efficacy in Breast Cancer: A Prospective Multicenter Trial. Clin Cancer Res 2018;24:2312-8.

5. Pistelli $M$, Mora AD, Ballatore $Z$, et al. Aromatase inhibitors in premenopausal women with breast cancer: the state of the art and future prospects. Curr Oncol 2018;25:e168-75.

6. Rutqvist LE, Cedermark B, Glas U, et al. Randomized trial of adjuvant tamoxifen combined with postoperative radiation therapy or adjuvant chemotherapy in 
postmenopausal breast cancer. Cancer 1990;66:89-96.

7. Fisher B, Dignam J, Wolmark N, et al. Tamoxifen and chemotherapy for lymph node-negative, estrogen receptorpositive breast cancer. J Natl Cancer Inst 1997;89:1673-82.

8. Morales L, Canney P, Dyczka J, et al. Postoperative adjuvant chemotherapy followed by adjuvant tamoxifen versus nil for patients with operable breast cancer: a randomised phase III trial of the European Organisation for Research and Treatment of Cancer Breast Group. Eur J Cancer 2007;43:331-40.

9. Aebi S, Sun Z, Braun D, et al. Differential efficacy of three cycles of CMF followed by tamoxifen in patients with ERpositive and ER-negative tumors: long-term follow up on IBCSG Trial IX. Ann Oncol 2011;22:1981-7.

10. Goldvaser H, Ribnikar D, Majeed H, et al. Absolute benefit from adjuvant chemotherapy in contemporary clinical trials: A systemic review and meta-analysis. Cancer Treat Rev 2018;71:68-75.

11. Pritchard KI. Combining endocrine agents with chemotherapy: which patients and what sequence? Cancer 2008;112:718-22.

12. Pico C, Martin M, Jara C, et al. Epirubicincyclophosphamide adjuvant chemotherapy plus tamoxifen administered concurrently versus sequentially: randomized phase III trial in postmenopausal node-positive breast cancer patients. A GEICAM 9401 study. Ann Oncol 2004; 15:79-87.

13. Albain KS, Barlow WE, Ravdin PM, et al. Adjuvant chemotherapy and timing of tamoxifen in postmenopausal patients with endocrine-responsive, node-positive breast cancer: a phase 3, open-label, randomised controlled trial. Lancet 2009;374:2055-63.

14. Bedognetti D, Sertoli MR, Pronzato P, et al. Concurrent vs sequential adjuvant chemotherapy and hormone therapy in breast cancer: a multicenter randomized phase III trial. J Natl Cancer Inst 2011;103:1529-39.

15. Poggio F, Ceppi M, Lambertini M, et al. Concurrent versus sequential adjuvant chemo-endocrine therapy in hormone-receptor positive early stage breast cancer patients: a systematic review and meta-analysis. Breast 2017;33:104-8.

16. Montemurro F, Perrone F, Geuna E. Adjuvant ovarian suppression in premenopausal breast cancer. N Engl J Med 2015;372:1672-3.

17. Francis PA, Pagani O, Fleming GF, et al. Tailoring Adjuvant Endocrine Therapy for Premenopausal Breast Cancer. N Engl J Med 2018;379:122-37.

18. Higgins JP, Welton NJ. Network meta-analysis: a norm for comparative effectiveness? Lancet 2015;386:628-30.

19. Furukawa TA, Salanti G, Atkinson LZ, et al. Comparative efficacy and acceptability of first-generation and secondgeneration antidepressants in the acute treatment of major depression: protocol for a network meta-analysis. BMJ Open 2016;6:e010919.

20. Saaiq M, Ashraf B. Modifying "Pico" Question into "Picos" Model for More Robust and Reproducible Presentation of the Methodology Employed in A Scientific Study. World J Plast Surg 2017;6:390-2.

21. Parmar MK, Torri V, Stewart L. Extracting summary statistics to perform meta-analyses of the published literature for survival endpoints. Stat Med 1998;17:2815-34.

22. Tierney JF, Stewart LA, Ghersi D, et al. Practical methods for incorporating summary time-to-event data into metaanalysis. Trials 2007;8:16.

23. Viswanathan M, Ansari MT, Berkman ND, et al. Assessing the Risk of Bias of Individual Studies in Systematic Reviews of Health Care Interventions. In: Methods Guide for Effectiveness and Comparative Effectiveness Reviews. Rockville (MD): Agency for Healthcare Research and Quality (US); March 8, 2012.

24. Woods BS, Hawkins N, Scott DA. Network meta-analysis on the log-hazard scale, combining count and hazard ratio statistics accounting for multi-arm trials: a tutorial. BMC Med Res Methodol 2010;10:54.

25. Higgins JP, Thompson SG. Quantifying heterogeneity in a meta-analysis. Stat Med 2002;21:1539-58.

26. Dias S, Welton NJ, Caldwell DM, et al. Checking consistency in mixed treatment comparison meta-analysis. Stat Med 2010;29:932-44.

27. Higgins JP, Thompson SG, Deeks JJ, et al. Measuring inconsistency in meta-analyses. BMJ 2003;327:557-60.

28. Hutton B, Salanti G, Caldwell DM, et al. The PRISMA extension statement for reporting of systematic reviews incorporating network meta-analyses of health care interventions: checklist and explanations. Ann Intern Med 2015;162:777-84.

29. Hackshaw A, Baum M, Fornander T, et al. Long-term effectiveness of adjuvant goserelin in premenopausal women with early breast cancer. J Natl Cancer Inst 2009;101:341-9.

30. Kaufmann M, Graf E, Jonat W, et al. A randomised trial of goserelin versus control after adjuvant, risk-adapted chemotherapy in premenopausal patients with primary breast cancer - GABG-IV B-93. Eur J Cancer 2007;43:2351-8.

31. Hubay CA, Pearson OH, Marshall JS, et al. Adjuvant chemotherapy, antiestrogen therapy and immunotherapy 
for stage II breast cancer: 45-month follow-up of a prospective, randomized clinical trial. Cancer 1980;46:2805-8.

32. Hata $Y$, Takahashi $H$, Todo $S$, et al. Ten-year results of a randomized trial on adjuvant chemo-endocrine therapy with tamoxifen for stage II breast cancer. Breast Cancer 2003;10:134-9.

33. Hutchins LF, Green SJ, Ravdin PM, et al. Randomized, controlled trial of cyclophosphamide, methotrexate, and fluorouracil versus cyclophosphamide, doxorubicin, and fluorouracil with and without tamoxifen for highrisk, node-negative breast cancer: treatment results of Intergroup Protocol INT-0102. J Clin Oncol 2005;23:8313-21.

34. Kim HA, Lee JW, Nam SJ, et al. Adding Ovarian Suppression to Tamoxifen for Premenopausal Breast Cancer: A Randomized Phase III Trial. J Clin Oncol 2020;38:434-43.

35. Regan MM, Walley BA, Francis PA, et al. Concurrent and sequential initiation of ovarian function suppression with chemotherapy in premenopausal women with endocrineresponsive early breast cancer: an exploratory analysis of TEXT and SOFT. Ann Oncol 2017;28:2225-32.

36. Schwartzberg LS, Wang G, Somer BG, et al. Phase II trial of fulvestrant with metronomic capecitabine for postmenopausal women with hormone receptor-positive, HER2-negative metastatic breast cancer. Clin Breast Cancer 2014;14:13-9.

37. Nakai M. Fulvestrant Combined with Capecitabine May Be Effective and Well Tolerated for Patients with Estrogen Receptor-Positive, Her2-Negative Metastatic Breast Cancer. Breast 2015;24:S50-1.

38. Li N, Hao Y, Kageleiry A, et al. Time on treatment of everolimus, fulvestrant, and capecitabine for the treatment of HR+/HER2-metastatic breast cancer: A retrospective claims study in the US. Cancer Res 2016. doi: 10.1158/1538-7445.SABCS15-P4-13-14.

39. Martin M, Kahan Z, Carrasco E, et al. Abstract OT201-06: Phase III study of palbociclib (PD-0332991) in combination with endocrine therapy (exemestane or fulvestrant) versus chemotherapy (capecitabine) in hormonal receptor (HR) positive/HER2 negative metastatic breast cancer (MBC) patients with resistance to aromatase inhibitors. "The PEARL study” (GEICAM/2013-02). Cancer Res 2017. doi:
10.1158/1538-7445.SABCS16-OT2-01-06.

40. Sutherland RL, Green MD, Hall RE, et al. Tamoxifen induces accumulation of MCF 7 human mammary carcinoma cells in the G0/G1 phase of the cell cycle. Eur J Cancer Clin Oncol 1983;19:615-21.

41. Mohammadianpanah M, Ashouri Y, Hoseini S, et al. The efficacy and safety of neoadjuvant chemotherapy +/letrozole in postmenopausal women with locally advanced breast cancer: a randomized phase III clinical trial. Breast Cancer Res Treat 2012;132:853-61.

42. Yu KD, Wu SY, Liu GY, et al. Concurrent neoadjuvant chemotherapy and estrogen deprivation in patients with estrogen receptor-positive, human epidermal growth factor receptor 2-negative breast cancer (CBCSG-036): A randomized, controlled, multicenter trial. Cancer 2019;125:2185-93.

43. Early Breast Cancer Trialists' Collaborative Group (EBCTCG); Davies C, Godwin J, et al. Relevance of breast cancer hormone receptors and other factors to the efficacy of adjuvant tamoxifen: patient-level meta-analysis of randomised trials. Lancet 2011;378:771-84.

44. Pusztai L. Molecular heterogeneity of breast cancer: implications for treatment and clinical trial design. Breast Cancer Res 2009;11:S4.

45. Felding-Habermann B, O'Sullivan DM, Lorger M, et al. Breast Cancer Heterogeneity and Treatment Resistance: Clues from Metaplastic Tumors. Cancer Res 2011. doi:10.1158/0008-5472.SABCS11-PD03-07.

46. Alix-Panabières C, Pantel K. Abstracts from the 11th International Symposium on Minimal Residual Disease, 3-5 May 2018, Montpellier, France. Clin Exp Metastasis 2018;35:167-217.

47. Jonchère $B$, Vétillard $A$, Toutain $B$, et al. Contribution to tumor escape and chemotherapy response: A choice between senescence and apoptosis in heterogeneous tumors. Bull Cancer 2016;103:73-86.

doi: $10.21037 /$ tbcr-21-3

Cite this article as: Li T, Shan Z, Shi Y, Kuang X, Yu L, Tang SC, Shao N, Lin Y. Sequential versus concurrent adjuvant chemo-endocrine therapy for HR+ early breast cancer: a systematic review and Bayesian network meta-analysis. Transl Breast Cancer Res 2022;3:8. 